\title{
Presencia de síntomas somáticos en niños y niñas: una comparación entre el período sin exámenes y con exámenes
}

\author{
Presence of somatic symptoms in girls and boys: a \\ comparison between the period without examinations and \\ with examinations
}

\author{
Yuranis Stella Paternina-Martínez (iD \\ $\triangle$ yuranis.paternina@cecar.edu.co \\ Corporación Universitaria del Caribe-CECAR, Sincelejo, Colombia
}

Hailin Patricia Pretel-Contreras

Recibido: 06-09-2018

$\triangle$ hailin.pretel@cecar.edu.co

Corporación Universitaria del Caribe-Cecar, Sincelejo, Colombia

Aceptado: 11-12-2018

Publicado: 17-12-2018

\section{RESUMEN}

Objetivo. Comparar la presencia de síntomas somáticos durante un periodo de examen y uno sin examen en los colegios públicos y privados de la ciudad de Sincelejo. Método. El estudio es de enfoque cuantitativo, de tipo descriptivo comparativo, participaron 358 estudiantes, de los cuales 124 fueron mujeres (34,6\%) y 234 hombres $(65,4 \%)$ a los cuales se les aplicaron el inventario de somatización para niños. Resultados y Conclusiones. No se hallaron diferencias significativas entre los dos períodos estudiados, ni tampoco entre chicos y chicas. Se encontró, no obstante, que los diez síntomas más frecuentes son el dolor de cabeza, sensación de cansancio o baja energía, dolor en los músculos, debilidad en algunas partes del cuerpo, náuseas o malestar estomacal, dolor en el estómago o abdomen, latidos del corazón muy rápidos, sensación de vómitos, dolor en las rodillas, codos o articulaciones, y por último dolor en los brazos y piernas.

Palabras clave: Síntomas somáticos, ansiedad, estrés, examen finales.

\section{ABSTRACT}

Objective. To compare the presence of somatic symptoms during a period of examination and one without examination in the public and private schools of the city of Sincelejo. Methods. The study is quantitative in approach, descriptive in type, 358 students participated, of which 124 were women (34.6\%) and 234 men (65.4\%) to whom the inventory of somatization for children was applied. Results and Conclusions. No significant differences were found between the two periods studied, nor between boys and girls. It was found, however, that the ten most frequent symptoms are headache, feeling tired or low energy, muscle pain, weakness in some parts of the body, nausea or stomach discomfort, pain in the stomach or abdomen, very fast heartbeat, sensation of vomiting, pain in the knees, elbows or joints, and finally pain in the arms and legs.

Keywords: Somatic symptoms, anxiety, stress, final exam. 


\section{INTRODUCCIÓN}

Según De Gucht y Fischler (2002) los exámenes escolares se caracterizan por ser un fenómeno primario que genera diversos malestares tales como dolor de cabeza, problemas digestivos, aceleración del ritmo cardiaco y de la respiración, sudoración en las manos, problemas para dormir y pesadillas. El término "somatización" hace referencia a la presencia de manifestaciones orgánicas derivadas de un trastorno psíquico que provocan malestar en el individuo, las cuales, desde el punto de vista médico, no tiene un origen orgánico (Romero et al., 2013).

El trastorno de somatización cambia al trastorno de síntomas somáticos en el DSM-V. EI trastorno de síntomas somáticos es la presencia de síntomas físicos durante seis meses, los cuales desencadenan afectaciones en el funcionamiento del individuo, por ejemplo, preocupaciones, disgustos, sentimientos desagradables y conductas inapropiadas. En los niños también existe la presencia de síntomas somáticos, los más comunes son dolor abdominal, dolor de cabeza y nauseas; cabe resaltar que en los niños normalmente solo predomina un solo síntoma en comparación con los adultos (APA, 2013; DomenechLlaberia, Romero, Canals, Hernandez-Martinez, 2015).

Spielberger (1966) señala que la ansiedad que experimentan los estudiantes ante los exámenes tiene dos componentes. El primer componente es la preocupación, la cual se define como los pensamientos que se tienen sobre el fracaso y sus posibles consecuencias. Por lo tanto, la persona pierde la total concentración debido al surgimiento de pensamientos negativos. También se encuentra el componente emotivo, que consiste en sensaciones desagradables y reacciones fisiológicas tales como tensión, inquietud, molestia y desagrado. Además, este autor plantea que la ansiedad contribuye como un componente cognitivo debido a que, el sujeto visualiza anticipadamente las consecuencias negativas que puede generar el fracaso, lo que desencadena interferencias en su actividad cognitiva.

Sarason (1978) desarrolló una serie de estrategias con el objetivo de disminuir la conducta ansiosa en los estudiantes ante los exámenes. En primer lugar, recomienda que el alumno acepte la ansiedad para que posteriormente sea capaz de sustituir el rechazo, la rabia o el temor que pueda sentir por la aceptación del hecho que exacerba los síntomas. Asimismo, menciona reevaluar la amenaza percibida; esto consiste en que el alumno logre una visión más ponderada y real de la situación; otras de las pautas radican en analizar con el alumno los recursos que posee para enfrentar la situación. Además, proporcionarles a los estudiantes herramientas que les permitan confrontar el problema y reforzar sus habilidades para alcanzar el éxito. Si todas estas ideas se emplean se puede lograr minimizar la ansiedad en los estudiantes en la época de los exámenes.

Gutiérrez y Avero (1995) explican que al momento de presentar un examen escolar, los alumnos experimentan una elevada ansiedad, esto repercute no solo en la evaluación, sino en la vida escolar, lo que genera desequilibrios en la salud mental. Debido a la situación anteriormente mencionada se puede comenzar a padecer síntomas somáticos como dolores de cabeza, náuseas y vómitos, acompañados de ansiedad. 
Paternina-Martínez \& Pretel-Contreras - Presencia de síntomas somáticos en niños y niñas

Fernández (1990) divide la presencia de síntomas somáticos en tres áreas:

Ámbito físico: se altera el sueño y la alimentación, aparecen dolores en el estómago y dolor en la cabeza.

Ámbito de comportamiento: se presentan conductas inadecuadas tales como ver televisión en exceso.

Ámbito cognitivo: el estudiante infravalora o le da poca importancia al estudio, pero a la vez le preocupa qué dirán sus padres si pierde el examen.

Por lo general, en la sociedad colombiana los padres de familia y los docentes no se percatan de la presencia de malestares físicos y psicológicos que surgen dentro de la escuela debido a diferentes situaciones escolares: realización de los exámenes académicos, adaptación escolar, sentimientos de preocupación y temores que experimentan al pasar a otro ciclo de curso escolar.

Algunos estudios anteriores (Fernández, 1990; Viñas y Caparrós, 2000) indican que la situación estresora de los exámenes tiende a desarrollar en el escolarizado elevados niveles de ansiedad y estrés. En algunos estudiantes se presentan desequilibrio físico y psicológico como una consecuencia de la percepción negativa que tienen hacia la situación. Por lo tanto, el rendimiento académico también se ve afectado, los estudiantes presentan mucha dificultad para concentrarse en problemas complejos que son requeridos por la realización de la evaluación. Los síntomas se presentan al momento de estudiar y preparar el examen, como también en la ejecución.

Barrio, García y Ruíz (2006) afirma que en la presentación de exámenes se evidencia diferentes problemas emocionales tales como síntomas somáticos, ansiedad y estrés. Desde hace tiempo la psicología abarcó el término "estrés" como la sobrecarga de situaciones agobiantes y de gran importancia para los individuos, que ocasionan tensión y presencia de síntomas físicos.

Jadue (2001) y Bados (2005) afirman que otro problema emocional es la ansiedad que se presentan en los exámenes de forma relativamente frecuente y puede afectar hasta un $18 \%$ de los niños y adolescentes, lo que afecta el rendimiento académico, el estado de ánimo y se relaciona con la presencia de diversos síntomas somáticos los cuales generan en los niños frustraciones y poco interés escolar. En Estados Unidos, Campo y Fritsch (1994), en una muestra de la población general, encontraron que entre el $10 \%$ y $30 \%$ de los niños y adolescentes se encuentran afectados por los síntomas somáticos los cuales padecen al momento de la realización de actividades académicas de gran importancia.

Recientemente se llevó a cabo una investigación dirigida por Rodríguez, Dapia y López (2014) en la que se estudió la ansiedad ante los exámenes en alumnado de secundaria con una muestra de 325 estudiantes, entre los doce y diceciséis años de edad. Para la recogida de datos implementaron el cuestionario de evaluación de ansiedad ante los exámenes. Tal estudio arrojó que el $90 \%$ de los estudiantes presentan respuestas fisiológicas y el $73 \%$ respuestas de evitación. Por lo tanto, la investigación evidencia la presencia de índices de estrés, ansiedad, preocupación debido a que desencadenan síntomas físicos, que se manifiestan con frecuencia en el periodo de examen y en los primeros cursos. 
Teniendo en cuenta los anteriores hallazgos, se hace cada vez más necesario llevar a cabo la investigación, con el fin de conocer si los estudiantes de nueve a dieciséis años de edad de los colegios públicos y privados de la ciudad de Sincelejo, Colombia, presentan síntomas somáticos o no a la hora de presentar los exámenes escolares. Además, qué género posiblemente puede experimentar mayor sensación de síntomas somáticos; el masculino o el femenino. Este interrogante se generó debido a los aumentos de malestar por parte de los niñas y niños ante situaciones de mucha concentración en el colegio como los son los exámenes; también presentan otros síntomas como ansiedad y estrés, que ocasionan dolores físicos, náuseas y problemas gastrointestinales asociados a los síntomas somáticos.

Esta investigación tiene como objetivo general comparar la frecuencia de síntomas somáticos en un período de exámenes y en otro período sin exámenes en niños y niñas de nueve a dieciséis años.

\section{Método}

Tipo de investigación. Este estudio es de enfoque cuantitativo, de tipo descriptivo comparativo (Hernández, Fernández y Baptista, 2003).

Población y muestra. El presente estudio se llevó acabo en la ciudad de Sincelejo, departamento de Sucre. La elección de las instituciones fue de manera intencional. Estas fueron Santa Rosa de Lima, Normal Superior de Sincelejo, San Vicente de Paul, Policarpa Salavarrieta, San Antonio, Antonio Lenis, Simón Araujo, Madre Amalia, Liceo Manantial, Las Mercedes y Decroly. De cada colegio público y privado se escogió la muestra de los estudiantes de quinto de primaria. Participaron en total 358 escolarizados, en las dos fases de la investigación, de los cuales 124 fueron chicas y 234 chicos, pertenecientes en su mayoría al estatus socioeconómico medio y alto. La media de edad fue de diez años (SD: 2,0$)$. Toda esta información se puede ver de manera más detallada en la Tabla 1.

Instrumentos. Para la recolección de datos se aplicó el inventario de Children's Somatizaction Inventory Walker, Garber y Green, (1991). El cuestionario evalúa 35 síntomas somáticos no especificados, con el fin de medir la frecuencia y la intensidad de los síntomas somáticos en los niños, niñas y adolescentes, el cuadernillo cuenta con veinticuatro ítems. Este inventario fue validado en Colombia a través del proyecto interno de la Corporación Universitaria del Caribe (CECAR) junto con el Semillero de Psicología Clínica y de la Salud, en un proyecto de investigación denominado "The Impact of examination on Students', Parents' and Teachers' Depression \& Anxiety in Colombian children".

Procedimiento. El proyecto obtuvo el consentimiento del comité de ética de la CECAR. Se visitó a cada institución y se especificó el objetivo del estudio, los instrumentos a utilizar, las ventajas de participar en el proyecto, entre otras cosas. En los colegios en los que la respuesta fue afirmativa se procedió a entregar a los padres de familia el consentimiento informado para que autorizaran la participación de su hijo o hija en la investigación; seguidamente, al estudiante que estaba autorizado por su padre o madre se le aplicaba el instrumento. 
Paternina-Martínez \& Pretel-Contreras - Presencia de síntomas somáticos en niños y niñas

Análisis estadístico. En este análisis se calculó la media, la frecuencia, las desviaciones típicas y el cálculo de comparación de muestras relacionadas ( $T$ de Student), a través del programa estadístico para las ciencias sociales: Statistical Package for Social Science (SPSS), el cual ofrece un análisis completo de los datos.

Tabla 1. Datos sociodemográficos.

\begin{tabular}{ccc}
\hline & $\mathbf{n}$ & \% \\
\hline Sexo & & \\
Niño & 234 & 65,4 \\
Niña & 124 & 34,6 \\
Estatus socioeconómico & & \\
Medio-alto & 136 & 38,0 \\
Medio & 88 & 24,6 \\
Medio-bajo & 93 & 26,0 \\
Bajo & 23 & 6,4 \\
Edad & & \\
9 -12 años & 345 & 99,2 \\
$13-16$ años & 3 & 9 \\
\hline
\end{tabular}

\section{RESULTADOS}

Presencia de síntomas somáticos en chicos y chicas en época de examen y sin examen.

En la Tabla 2 se evidencian los diez primeros síntomas más frecuentes de la muestra como principal encontramos dolor de cabeza tanto en la época de examen y sin examen.

Tabla 2. Comparación de presencia de síntomas somáticos en época sin examen y en época de exámenes.

\begin{tabular}{|c|c|c|c|c|c|c|c|c|}
\hline & \multicolumn{4}{|c|}{ Primera fase } & \multicolumn{4}{|c|}{ Segunda fase } \\
\hline & \multicolumn{2}{|c|}{ Chicas } & \multicolumn{2}{|c|}{ Chicos } & \multicolumn{2}{|c|}{ Chicas } & \multicolumn{2}{|c|}{ Chicos } \\
\hline & $\mathbf{n}$ & $\%$ & $\mathbf{n}$ & $\%$ & $\mathbf{n}$ & $\%$ & $\mathbf{n}$ & $\%$ \\
\hline Dolor de cabeza & 69 & 55,6 & 157 & 67,1 & 63 & 50,8 & 116 & 49,6 \\
\hline Sensación de cansancio o baja energía & 71 & 57,3 & 109 & 46,6 & 58 & 46,8 & 94 & 40,2 \\
\hline Dolor en los músculos & 55 & 44,4 & 72 & 30,8 & 49 & 39,5 & 79 & 33,8 \\
\hline $\begin{array}{c}\text { Sensación de calor o frio } \\
\text { (sensaciones de calor o frio repentinas sin razón) }\end{array}$ & 29 & 23,4 & 57 & 24,4 & 36 & 29 & 52 & 22,2 \\
\hline Debilidad en algunas partes del cuerpo & 56 & 45,2 & 85 & 36,3 & 42 & 33,9 & 65 & 27,8 \\
\hline Náuseas o malestar estomacal & 39 & 31,5 & 79 & 33,8 & 35 & 28,2 & 58 & 24,8 \\
\hline Dolor en tu estómago o abdomen & 53 & 42,7 & 102 & 43,6 & 34 & 27,4 & 73 & 31,2 \\
\hline Sensación de vómito & 32 & 25,8 & 73 & 31,2 & 29 & 23,4 & 56 & 23,9 \\
\hline Dolor en tus rodillas, codos o articulaciones & 45 & 36,3 & 71 & 30,3 & 34 & 27,4 & 71 & 26,1 \\
\hline Dolor en los brazos y piernas & 61 & 49,2 & 99 & 42,3 & 51 & 41,1 & 99 & 142,3 \\
\hline
\end{tabular}


Diferencia de género en la presentación de síntomas somáticos. En la Tabla 3 se puede apreciar que no hay diferencia estadísticamente significativa entre chicos y chicas en la presentación de síntomas somáticos en el periodo de examen. Las chicas presentaron mayor frecuencia de síntomas somáticos en comparación con los chicos, pero la diferencia no es significativa.

Tabla 3. Diferencia de género.

\begin{tabular}{ccccccc}
\hline & Género & N & Media & $\begin{array}{c}\text { Desviación } \\
\text { estándar }\end{array}$ & $\begin{array}{c}\text { Media de error } \\
\text { estándar }\end{array}$ & $\boldsymbol{P}$ \\
\hline Síntomas somáticos Sin & Chicas & 124 & 10,5645 & 11,55773 & 1,03791 & 0,288 \\
exámenes & Chicos & 234 & 9,4359 & 10,52027 & 0,68773 & \\
Síntomas somáticos con & Chicas & 121 & 9,9008 & 14,32562 & 1,30233 & 0,351 \\
exámenes & Chicos & 232 & 8,4569 & 10,75617 & 0,70618 & \\
\hline
\end{tabular}

\section{DISCUSIÓN}

Se encuentran diferentes postulados con similitudes acerca de la definición de "síntomas somáticos". Dimsdale (2018) dice que el trastorno de síntomas somáticos se caracteriza por la presencia de múltiples síntomas físicos que pueden ser acompañados o no de una enfermedad médica conocida; el diagnóstico del trastorno de síntomas somáticos se basa en antecedentes familiares del paciente.

Las personas que padecen trastornos somatomorfos o síntomas somáticos no fingen sus síntomas, pues los experimentan o los sienten como reales. Exista o no una explicación médica, estas personas experimentan un gran malestar o interferencia en su vida cotidiana a causa de los síntomas somáticos como también de otros trastornos que se evidencian a diario; los pacientes que presentan estos síntomas acuden con gran frecuencia a atención primaria y otros servicios sanitarios mientras que la asistencia a salud mental es menos (García, 2018).

En la actualidad existen pocas investigaciones sobre el tema de síntomas somáticos, lo cual genera que este tema sea desconocido para muchas personas y no se lleve a cabo una intervención que ayude tanto a niños como a jóvenes a disolver esta problemática que de algún modo tiene sus consecuencias en la vida personal e interpersonal de quien padece estos síntomas, debido a que se puede presentar en cualquiera persona sin importa la edad y género. En cualquiera situación de nuestro vivir se pueden padecer síntomas somáticos, es por eso que surge la necesidad de estudiarlos en niños, niñas y adolescentes tanto en la época de examen como sin examen, porque es una de las situaciones en que el estudiante puede presentar una sobrecarga emocional con respecto a la responsabilidad que tiene ante esta responsabilidad y meta. Este problema se desarrolla debido a la presión en la que se encuentra el estudiante al momento de presentar un examen escolar, pero algunos padres de familia y docentes son los que ejercen mayor presión ante esta situación, y todo es porque está relacionado con al buen rendimiento académico, en el cual se espera que el alumno tenga un buen desempeño, pero ellos a su vez desconocen que los escolarizados pueden experimentar síntomas somáticos y otros problemas en la salud metal que afecten su desempeño escolar y sus calificaciones. 
El objetivo general de la investigación es comparar la frecuencia de síntomas somáticos con examen y sin examen en niños y adolescentes de nueve a dieciséis años. El presente estudio es el primero en desarrollarse en el Departamento de Sucre. Los resultados de esta investigación muestran que existe presencia de síntomas somáticos en los estudiantes tanto en la época de examen y sin examen, los diez síntomas más frecuentes que experimentan los escolarizados son: dolor de cabeza (como principal), seguido de sensación de cansancio o baja energía, dolor en los músculos, debilidad en algunas partes del cuerpo, náuseas o malestar estomacal, dolor en el estómago o abdomen, latidos del corazón muy rápidos, sensación de vómitos, dolor en las rodillas, codos o articulaciones y, por último, dolor en los brazos y piernas. También se puede decir que no se encontró una diferencia significativa en cuanto al género; es decir que tanto varones como mujeres presentaron síntomas por igual.

Los resultados encontrados en este estudio guardan similitud con el estudio ejecutado por Serra (2012) en el cual el objetivo era determinar la prevalencia de los síntomas somáticos en niños de primaria ante los exámenes; alli se halló evidencia de síntomas somáticos en los niños y el dolor abdominal 36\% como principal síntoma, seguido de dolor de cabeza $25,9 \%$, dolor en las piernas $17,9 \%$, cansancio $17,4 \%$, mareos $3,5 \%$ y otros síntomas 10,1\%. La relación de estos estudios radica en la existencia de diferentes síntomas somáticos los cuales fueron los mismos encontrados en ambas investigaciones. Otras investigaciones se han centrado en estudiar detenidamente el tema de síntomas somáticos, cada una con énfasis en diferentes aspectos, pero guardan coincidencia en la aparición de estos síntomas. Por ejemplo, Viñas y Caparros (2000) dan a conocer en sus evidencias la prevalencia de síntomas somáticos en estudiantes. En la investigación de estos autores se buscaba analizar las estrategias que utilizaban los estudiantes para afrontar la época de examen y asimismo conocer los síntomas somáticos que experimentaban. Estos autores lograron determinar que los estudiantes que adoptan una posición positiva y activa ante los exámenes tienden a ser menos propensos a experimentar síntomas somáticos durante la participación de actividades académicas como son los exámenes escolares.

Es importante señalar estudios como el de Rodríguez et al., (2014), el cual se basó en la recolección de respuestas motoras, verbales, cognitivas o fisiológicas que pueden presentar los niños al momento de realizar un examen. Los resultados obtenidos fueron los siguientes: un $25,2 \%$ presenta un nivel de ansiedad bajo mientras que 243 estudiantes $(74,7 \%)$ tienen ansiedad media-alta. Aunque el método de recolección de los datos de esta investigación tiene mucha diferencia con la metodología del presente estudio, guarda relación con los resultados encontrados ya que se evidencia ansiedad y esta se presenta en los estudiantes acompañada de síntomas somáticos.

Finalmente, es necesario apreciar la necesidad de estudiar los síntomas somáticos teniendo en cuenta diferentes muestras y situaciones, con el fin de aumentar la cifra con respecto a investigación sobre síntomas somáticos, ya que en la actualidad existe escasez de estudios acerca de este tema.

\section{Conclusiones}

Luego de la finalización de la investigación y del respectivo proceso de interpretación de los resultados, se llegó a la conclusión de que existe la presencia de síntomas somáticos en los niños y niñas de quinto de primaria, tanto en la época de examen como fuera de 
ella, sin embargo, no existen diferencias significativas entre la presentación de síntomas en el período de exámenes y el período sin exámenes. No obstante, ahora se sabe que los diez primeros síntomas con más frecuencia son dolor de cabeza, sensación de cansancio o baja energía, dolor en los músculos, debilidad en algunas partes del cuerpo, náuseas o malestar estomacal, dolor en el estómago o abdomen, latidos del corazón muy rápidos, sensación de vómitos, dolor en las rodillas, codos o articulaciones y, por último, dolor en los brazos y piernas. Además, también se halló que no existe diferencia significativa en la presentación de síntomas somáticos entre chicos y chicas.

\section{Referencias}

American Psychiatric Association (APA); Kupfer, D.J.; Regier, D.A.; Arango López, C.; Ayuso-Mateos, J.L.; Vieta Pascual, E. y Bagney Lifante, A. (2014). DSM-5: Manual diagnóstico y estadístico de los trastornos mentales, 5a ed. Madrid: Editorial Médica Panamericana. Recuperado de: https://psicovalero.files.wordpress.com/.../manualdiagnc3b3stico-y-estadc3adstico-de.

Bados, A. (2005). Trastorno de ansiedad por separación. Rechazo escolar y fobia escolar. Facultad de Psicología. Universidad de Barcelona. Recuperado de: http://diposit. ub.edu/dspace/bitstream/2445/354/1/119.pdf

Barrio, J.; Garcia, M. y Ruiz, I. (2006). El estrés como respuesta. Revistas científicas de América Latina, el Caribe, España, Portugal,1, 37-48. Recuperado de: http://www. redalyc.org/pdf/3498/349832311003.pdf

Bausela, E. (2005, agosto 08). Ansiedad ante los exámenes: Evaluación e intervención psicopedagógica. Recuperado de: https://www.researchgate.net/profile/Esperanza Bausela Herreras/publication/28109768 Ansiedad ante los examenes evaluacion e intervencion psicopedagogica/links/5630e52b08ae506cea675cb9. pdf

Campo, J. y Fritz, G. (2007). Lewis's child and adolescent psychiatry a comprehensive textbook. Philadelphia: Lippincott William \& Wikins. Recuperado de: https://www. jaacap.org/article/S0890-8567(09)62295-4/pdf

De Gucht, V. y Fischler, B. (2002). Somatization a critical review of conceptual and methodological issues. Psychosomatics, 43, 1-9. Recuperado de: https://www.ncbi. nlm.nih.gov/pubmed/11927751

Dimsdale, J. (2018). Aspectos generales de la somatización. Manual Merck.

Domenech-Llaberia, E. ; Romero, K.; Canals, J. y Hernández-Martínez, C. (2015). Somatic symptoms in a Spanish child and adolescent school population. European Child \& Adolescent Psychiatry, 20(1), S195-S195.

Fernández, L. (1990). Cambios conductuales y su efecto sobre las puntuaciones en los exámenes. Revista Psicológica, 11, 1-18.

García, A. (6 de marzo del 2018). Trastorno somatomorfo o trastorno de síntomas somáticos - Una guía útil. [Cognifit blog]. Recuperado de https://blog.cognifit.com/ es/trastorno-somatomorfo-sintomas-somaticos/ 
Gutiérrez Calvo, M. y Avero, P. (1995) Ansiedad, estrategias auxiliares y comprensión lectora: Déficit de procesamiento versus falta de confianza. Psicothema, 7, (3), 569578. Recuperado de http://www.psicothema.com/pdf/1003.pdf

Hernández, R., Fernández, C., y Baptista, P. (2013). Metodología de la investigación. Recuperado de: http://catarina.udlap.mx/u_dl_a/tales/documentos/lad/blanco_s_ $\mathrm{nl} /$ capitulo3.pdf

Jadue, G. (2001). Algunos efectos de la ansiedad en el rendimiento escolar. Estudios Pedagógicos, 27, 111-118. Recuperado de: http://www.scielo.cl/scielo. php?script $=$ sci arttext $\&$ pid $=$ S0718-07052001000100008

Rodríguez, A. ; Dapia, D. y López, A. (2014). Ansiedad ante los exámenes en alumnado de secundaria obligatoria. Revista de estudio e investigación en psicología y educación, 1, 132-140. Recuperado de: http://revistas.udc.es/index.php/reipe/article/view/ reipe. 2014.1 .1 .14

Romero-Acosta, K.; Canals, J.; Hernández-Martínez, C.; Penelo, E.; Zolog, T.C. y Domènech-Llaberia, E. (2013). Age and gender differences of somatic symptoms in children and adolescents. Journal of Mental Health, 22(1); 33-41. Recuperado de: $\quad$ https://www.researchgate.net/publication/235365778 Age and Gender Differences of Somatic Symptoms in Children and Adolescents

Sarason, I. (1978). Personalidad. Un enfoque objetivo. México: Ed. Limusa.

Serra, R. (2013) Síntomas somáticos funcionales en una muestra española: psicopatología y estilos educativos. Revista anales de pediatría, 79, 2, 65-132.

Spielberger, C.D. (1966). Theory and research on anxiety. En C.D. Spielberger (ed.). Anxiety and Behavior (pp. 3-22). Nueva York: Academic Press.

Viñas, F. y Caparrós, B. (2000). Afrontamiento del periodo de exámenes y sintomatología somática autoinformada en estudiantes universitarios. Revista Electrónica dePsicología, 4(1). Recuperado de: https://es.scribd.com/document/49455134/Afrontamientodel-periodo-de-examenes-y-sintomatologia-somatica-autoinformada-en-un-grupode-estudiantes-universitarios

Walker, L.S., Garber, J., y Green, J.W. (1991). Síntomas de somatización en pacientes pediátricos con dolor abdominal: relación con la cronicidad del dolor abdominal y la somatización de los padres. Revista de psicología infantil anormal, 19, (4), 379-394. 